purgative treatment is followed, while it also occurs in the routine methods of injections or irrigations. Not in frequently a new case appears for treatment with epididy. mitis as the first symptom of any malady that the patien has apparently noticed. This complication is the one which makes the period before recovery much longer. In my last series of cases the frequency of its occurrence lus fallen considerably. In my experience epididymitis is uulikely to occur except in the acute or subacute stages.

\section{Number of Treatments.}

In the absence of any complications twelve treatments are needed in an acute case. This means that electrical treatment is not required after the third week. By a survey of the last five years of this work this period has been getting shorter, and the present is not likely to spell timality. In this series of 500 cases 287 were acute and 213 chronic. The chronic cases were usually sent to me after not less than two months of treatment under the care of the patient's medical man. Either irrigation or injections had given some relief but had not arrested the disease, or there had been a relapse after ceasing treat ment. In such cases there needs to be microscopic examination as to the presence or not of gonococci. By this means one knows whether the condition is one of gleet.

For the reasons already given there should not be too fiequent use of electrolysis in these cases. In spite of local lavage that has been tried in the methods mentioned and perhaps vaccine and medicines, there still is a sup purative condition. The patient's resistance is weak, and if continued with no perceptible improvement one sees too often how irrigation leads to irritation Electrolysis three times weekiy, with no other local treatment, soon produces the desired cliange, and so far I have not met the case which will not yield to careful assistance given this way provided the patient co-operates in the programme laid down. Someimes the patient's failure to recover under his doctor's care is due to the patient's own impatience or to indulgences of the alcololic or other types. Sometimes also he has been disappointed by the unrealized promises of recovery within a period. The promise of recovery in a certain time is, of course, a factor in the patient's contidence at the beginning. I think, however, with the late Dr. Gee, that in most maladies prognosis is the most difficult of the informations expected of us. But as that gieat teacher used to remind his students, "Gentlemen, you will usually be wrong.'

In view of the immunity from rheumatic complications described, I believe the protection is due to the production of a specific antibody, probably allied chemically to gonococcal vaccine. If so, the vaccine I am making in a special way may, by itself, prove of greater value than existing forms, but I have not yet sufficient experience of it to make a detailed publication at the present stage. In curing gonorrhoea we are not able by any single operation to empty the mucous glands and follicles of the urethra of the infecting bacteria. A stream of liquid injected or irrigated does something in that direction, but if it could wash out the gonococci in those crypts we could remove them while the patient waits, so to speak. Manifestly we can no more do that than we can wash the infecting microbe out of the parotid gland (in a case of mumps) by irrigations through its duct. Treatment of gonorrhoea does, of course, assist recovery, and it does so by local germicidal action, by elevation of the resistance by the appropriate vaccine, and by the bactericidal action of urinary antiseptics. A good treatment therefore nust avoid washing away the precious antibodies which are in the lymph-bathed but inflamed parts, and at the same time it sliould help by destroying or otherwise removing from the affected channels the populations of the gonococci, whether dead or alive.

ACCORDING to recent statistics Vienna has lost 10 pe cent. of its population since 1910, or 190,000 inhabitants, 130,00 ab f whom were children or adolescents.

Professor EMILE JEANBRAU has been appointed the first professor of urology at the school of medicine at Montpellier. He was president of the twenty-first congress of the French Association of Urology recently held at Strasbourg.

\section{THE TRE.ITMENT OF NEGLECTED CASES} OF CLUB-FOOT.*

BY

W. PAYNTER NOALL, M.S.LOND., F.R.C.S.ENG., SURGEON, YORK COUNTY HOSPITAL.

THE commonest deformity resulting from infantile para. lysis is club-foot, the variety known as talipes equino-varus being the most frequent. This condition, as time elapses, becomes more pronounced, and, unless appropriate means are adopted in its early stage to correct the deformity, very radical procedures are necessary for its amelioration.

During the last four years I have had several cases of the different varieties of club-foot under my care which bave not had efficient treatment, some of them having been entirely negleeted. Their ages have varied from 7 years up to the twenties when first brought under observation. They have comprised cases of talipes equinovarus (paralytic and spastic varieties), talipes equinovalgus, talipes ca!caneo cavus, and talipes plantaris.

As pointed out by Rowlands, ${ }^{1}$ it is useless to expect that in the later stages tenotomies, with division of contracted ligaments and fascia, will alone rectify the deformity. The bones of the foot have grown and developed in an abnormal position, and it is this abnormal growth of the bones which proves an effective obstacle to the restoration of the foot to a normal shape. Rowlands advocates an operation througin the medio-tarsal joint, which is tantamount to an excision of this joint. I have followed closely on the same lines.

Talipes Equino-varus.

This deformity, when well developed, presents the following points of interest:

The affected limb is shorter than its fellow; there is wasting, which may be very marked; the leg feels cold, pre sents a bluish discoloration; the heel is raised from the ground; the foot is inverted and adducted. The head of the astragalus forms a marked projection on the dor sum of the foot. There is some degree of scoliosis (compensatory), which disappears on the patient being held up. The gait is awk. ward, the patient walking on the outer border of the foot, and where this comes in contact with the ground a callosity develops. There may, in addition, be some degree of rotation inwards of the lower part of the leg.

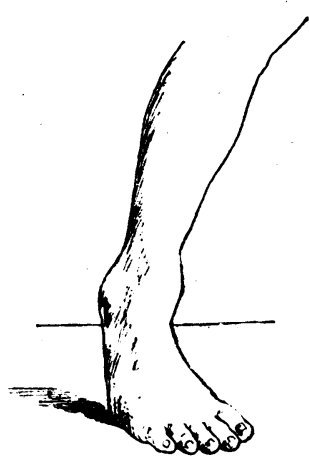

FIG. 1.-Talipes equinovarus.
Treatment.-Following the example of Sir Robert Jones, the operation is bloodless. After elevation of the limb a tourniquet is applied around the thigh so as to compress the femoral artery. Though numerous vessels are severed, there is $n n$ bleeding to obscure the field of operation, and consequently no time is taken up in tying vessels or $\mathrm{s}$ wabbing the wound.

The plantar fascia is put on the stretch, the tense bands being divided subcutaneously. The inferior and internal portions of the astragalo-scaphoid capsule and the long and short plantar ligaments are then divided. The foot is unrolled and stretched manually as much as possible. A curred incision is then made from below and in front of the internal malleolus, downwards and outwards over the dorsum of the foot towards its outer border. 'This incision is planned so as to open later the medio-tarsal joint, which latter is made up posteriorly of the head of the astragalus and fore part of the os calcis, articulating respectively with the scaphoid and cuboid anteriorly, each joint having a synovial cavity and being surrounded by a strong ligamentous capsule.

The incision is deepened, cutaneous nerves and reins are cut througli and then the anterior annular ligament of the ankle-joint is divided. The following tendons are exposed from within outwards: the tibialis anticus, extensor longus lallucis, extensor longus digitorum, and the peroneus tertius. These are freed and lifted forwards by means of a couple of hooks. The termination of the 
anterior tibial artery, vein and nerve are exposed between the tendons of the extensor longus digitorum and extensor longus hallucis. The nerve to the extensor brevis digitorum is, if possible, preserved, this latter muscle being detached from its origin from the os calcis and turned outwards The prominent head of the astragalus is easily made out and the astragalo-scaphoid joint opened. The periosteum and ligamentous capsule are separated with a rugine in a backward direction from the neck of the astragalus, and the head and part of the neck removed with a sharp chisel. At the age of 8 or 9 years this can be done with a chisel alone, but if the patient is much older a mallet will have to be used as we!l. .

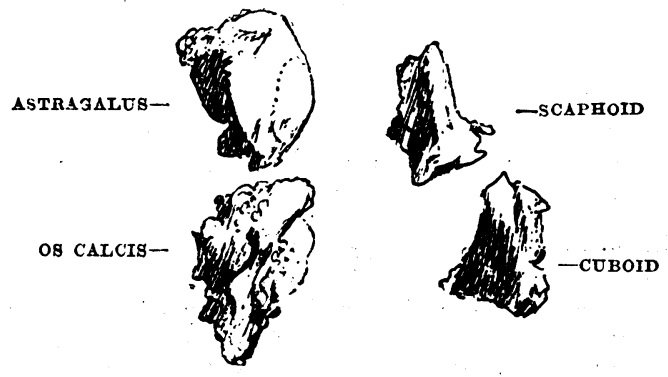

Frg. 2.-Parts removed at operation.

A thin layer is taken off the adjacent surface of the scaphoid. The calcaneo-cuboid joint is now opened dorsally and adjacent portions of these bones removed. In removing the head of the astragalus due regard must be had to the line of section, the direction being from above downwards and slightly forward and outwards. Sufficient amount of the bones is removed so as to eliminate the varus; the opposing surfaces should now lie snugly opposed in the corrected position. The medio-tarsal joint is now closed dorsally with stont chromic catgut sutures.

In cases where the tibialis anticus is strongly acting its insertion may be detached and sutured to the periosteum and ligamentous structures over the dosal surface of the cuboid so that it becomes an inverter of the foot. The origin of the extensor brevis digitorum is now sutured back in position to the upper surface of the os calcis. The annular: ligament is united over the extensor tendons and the skin closed. with interrupted silkworm gut sutures. The limb is now held vertically and a longitudinal incision made on the inner border of the tendo Achillis. This tendon is exposed and lengthened by a Z-shaped incision, the tendon being first slit longitudinally and then each half divided transversely, one half being divided above and the other half below. By sliding the two divided halves on each other the tendon can be lengthened to the required degree.

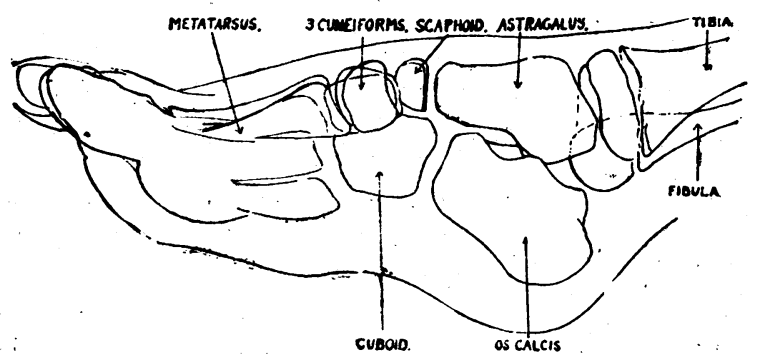

FIG. 3.-From a skiagraph of the foot before operation.

The posterior ligament of the anlile-joint is then divided, and the foot can now be dorsiflexed beyond a right angle, the equinus position being over-corrected. The two ends of the tendo Achillis are now sutured together with chromic catgut and the skin incision closed with inter. rupted silkworm gut sutures. Thie sutured lines are now painted with iodine and gauze dressings applied. The limb is surrounded with a layer of wool from above the knee to the base of the toes and a flannel bandage applied over all. While the foot is held in an over-corrected position the limb from above the knee to the toes. which are left free, is encased in plaster-of-Paris bandages. Before the casing has set it is slit up in its whole length anteriorly with a sharp scalpel and surrounded, with a single layer of cotton-wove bandage. The tourniquet is now removed. The circulation is now seen to be restored to the toes and the patient is returned to bed.

For the next forty-eight hours it is not uncommon for the patient to complain of some degree of pain in the limb; this is not accompanied by any sivelling of the toes or signs of haemorrhage, and need not cause any alarm. It is easily assuaged by the exhibition of a few minims of nepenthe, and passes off within two days.

The plaster is removed at the end of six weels. This is quite an easy matter, as it has already been slit up before leaving the operating table. 'The sutures are now removed and the limb replaced in its plaster casing. In a few days massage is commenced, and in the course of a month the patient is measured for boots and leg irons. Whilst these

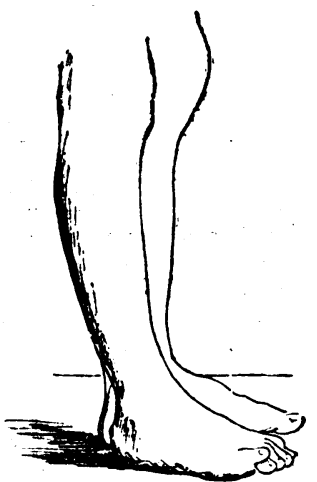

Fig. 4.-The foot nine weeks after operation. are being made the plaster casing serves as a splint to keep the foot in position. During the early part of the massage treatment, or on putting ihs foot to the gro ind, the patient may complain of pain in the sole, but this soon disappears. After the plaster is first removed there is no voluntary movement, but under massage this soon returns, and movements which before operation were feeble or even absent may become respectively stronger and apparent.

In cases of equino - varus, in provid̄ing leg irons (external and in. ternal), it is necessary to have a stop at the ankle to limit plantar flexion a $\mathrm{nd}$ permit dorsiflexion, an d, in addition, 8 varus T-strap to counteract any tendency to a return of the varus posi

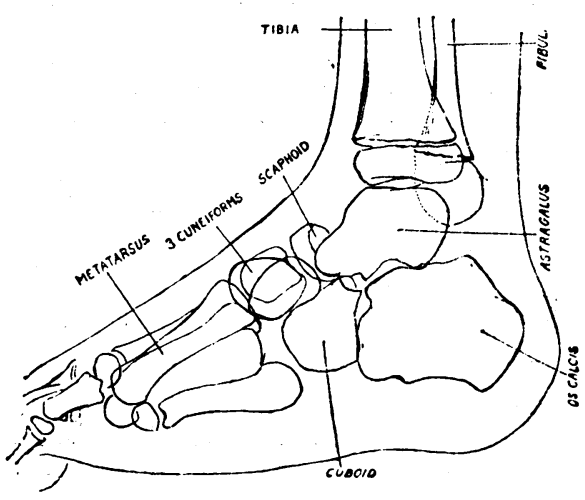

FIG. 5.-The foot after operation. tion. A cork sole is added inside the boot of a sufficient depth to compensate for the shortening of the limb. Where there is some inward rotation in addition of the lower part of the leg, this can be corrected by carrying the leg iron up to the pelvis, to which is fitted a pelvic band. Massage will still have to be continued and the apparatus worn till deformity does not tend to reappear and the limb become as useful as possible. As time goes on it will be found necessary to diminish the depth of the cork sole.

\section{Talipes Calcaneo-cavus.}

In this uncommon deformity, the opposite to equinovarus, the patient walks on the back of the heel and the fore part of the foot is dropped - that is to say, the portion of the foot in front of the medio-tarsal joint. I have had two cases of this deformity where the paralysis has not been complete, but where both the anterior and posterior group of muscles of the foot have exhibited some degree of movement. The operation in these cases has followed for the most part the one designed by Sir Robert Jones ${ }^{2}$ and quoted by Tubby. . It is divided in to two stages.

Stage 1.- 'Through an incision on the inner and outer border of the foot opposite the summit of the cavus deformity-that is, opposite the medio-tarsal joint-a wedge-shaped piece of bone, base upwards, is removed, transversely from the tarsus, the incisions are closed Iwith silkworm-gut sutures and the foot bandaged to the tibia with plaster-of-Paris bandages. If there is any varus the wedge removed is broader externally than internally, and if there is any valgus the $w \in d g e$ is broader internally. 
This operation corrects the cavas deformity, In four to five weeks the plaster is taken off and the sutures removed.

Stage 2.-At this operation the calcaneus deformity is corrected, the foot being brought down to a right angle. The anterior ligament of the anklc-joint is divided through an open incision across the front of that joint, the tendons being held out of the way. Through a longitudinal incision over the tendo Achillis the tendon is divided and a wedge-shaped piece of bone removed from the back of the astragalus, the base backwards, the superior articular surface of the astragalus being left intact so that we can still get dorsiflexion and pluntar flexion at the ankle-joint.

The foot is now brought into an over-corrected position, a sufficient portion of the tendo Achillis being removed so as to meet in this corrected position. The posterior incision is closed. In one of the cases a wide gap was left at the site of the anterior incision, and it was found necessary in order to close it to make a transverse incision above the ankle, joint, undercut its edges, and unite it vertically. Dressings are applied and the foot and leg put in plaster-of-Paris bandages. These are removed after six weeks, and the after-treatment is the same as indicated in cases of talipes equino-varus. Boots and irons are provided with a stop at the ankle to allow plantar flexion and limit dorsiflexion. A varus or valgus T-strap is added if there is any tendency to eithe: varus or valgus respectively.

\section{Talipes Plantaris.}

This condition--" hollow foot"-is bilateral, and variously supposed to be due to wearing short boots or to a neuritis affecting the extensor group of muscles of the foot following some of the iafectious fevers in childhood. 'The arch of the foot is higher than normal and the sole hollow, the toes are contracted, hyperextended at the metatarso. phalangeal joint and flexed at the interphalangeal joint, the structures in the sole of the foot are shortened. 'The foot cannot be flexed to or beyond a right angle. Corus develop on the heel and ball of the foot, walking being very painful, this being the chief symptom calling for treatment. This coudition can be treated on the same lines as outlined for talipes equino-varus.

\section{Results of Operation.}

By observing these cases over a number of years one finds that the paralysed limb, as a result of the operation, not only grows in length and girth, but it grows in length at a quicker rate than the sound limb. I have frequently observed cases where the affected limb has been from $1 \frac{1}{2}$ in. to 3 in. shorter than its fellow, that this amount of shortening decreases as time goes on, and in one case operated on four years ago, where the shortening was $1 \frac{1}{2}$ in., has to-day become practically nil, the gait is indistinguishable from that of a normal person, and the the patient has discarded all apparatus.

Table of some Cases where Measurements have been Recorded.

Talipes equino-varus. Operation, July 18th, 1917. Amount of shortening : November, 1917, $1 \frac{1}{3}$ in.; December, 1918, 5/8 in.; to-day, $1 / 4$ in.

Talipes equino-valgus. Operation, 1918. Amount of shorten. in : October, 1918, 11 in.; February 25th, 1921, 3/4 in.

ing: October, 1918, 11 in.; February 25th, 1921, 3/4 in. A of shortening: November, 1919, 3 in.; March, 1920, $1 \frac{1}{2}$ in. February, 1921, 1 is in.

Talipes calcaneo-cavus. Operation : first stage. March 19th, 1920; second stage, May lst, 1920. Amount of shortening: 1920, 1. in.; after operation, June, $1921,5 / 8$ in.

Talipes equino-varus. Operation, November, 1919. Amount of shortening: June 15th, 1920, 1/2 inch; February 24th, $1921,1,4$ in.

In sbme cases, where the paralysis is extreme, the patient may have to continue to wear the apparatus, but even in these cases the deformity has been corrected, the gait becomes more that of a normal person and léss of an eye. sore to its parents and friends, and can partalse more in the activities of life. A point to be noted in two cases of spastic talipes equino-varus (right-sided hemiplegia) is that the upper limb markedly improved after the foot. had been operated on.

In : undertaking the treatment of these cases it is neces. sary to have thrm under one's own supervision for a few years, also to have at one's command the services of a Bkilled masseuse, and to have provided correct boots and irons. Messrs. C.F. Thackray, of Leeds, have provided me with efficient apparatus.

The line drasvings from photographs and skiagram appended illustrate a case of talipes equino-varus before and after operation.

REFERENCES,

${ }^{1}$ R. P. Rowlands: Deformities, Choyce's Suroery. vol. iii. ${ }^{2}$ A. F Tubby: Deformities, including Diseascs of Bones and Joints, vol. i Tubby and Jones: Surgery of Paralysis.

\section{PIGMENTATION OF THE VERMIFORM APPENDIX. \\ (A Preliminary Note.)

\author{
E. M. COWELL, D.S.O., M.D., F.R.C.S.EvG.,
} LONDON.}

Thr literature on the subject of pigmentation of the vermiform appendix is at present scanty. In fact, a recent search through the various available indices of medical books and periodicals proved negative. No edition of the large monographs on appendicitis is of late enough date to include this subject.

This interesting pathological condition was first dis. covered a few years ago by W. H. Battle, ${ }^{1}$ and the results of a study of six of his specimens were published by Professor S. G. Shattock in 1916.2 In this article the naked-eye appearances and histological characteristics of the specimens are fully described, and possible explanations of the phenomena discussed. The diawing published by Shattock shows a mottled black pigmentation of the mucosa. The irregularity of the coloration is due to a sprinkling of light areas, corresponding to the situation of the lymphatic nodules. In the specimen removed from the case described in this paper the patchy distribution of the pigment was remarkable, reminiscent of the markings on the back of a snake. On analysis Professor Shattock failed to find any traces of iron in these specimens. He was also able to show that the pigment was not derived from blood. Histologically the pigment lies in the tissue surrounding the glandular crypts and is carried in large endothelial cells, all of which are superficial to the muscularis mucosae.

The subject is at present being actively investigated by Professor Sir Arthur Keith. In a recent demonstration at the Royal College of Surgeons he sljowed several sections of pigmented appendices. The granules are contained in large endothelial cells lying round the bases of the crypts of Lieberkühn. In sections taken from the colon in chronic cases of intestinal stasis these cells are being attacked by large numbers of phagocytes. The actual pigment seems to be related to melanin, and contains a fatty acid which is in some way allied to adrenaline. The changes that occur in the structure of the colon in chronic intestinal stasis are profound, but one of the most interesting is certainly the pigmentation of the raucosa.

In this connexion McCarrison's ${ }^{8}$ recent work on deficiency diseases is illuminating. This observer has been able to produce experimentally, by feeding animals on a vitamin-free diet, changes in the bowel similar to those described by Iieith. While no mention is made of the aupendix, the mucous membrane of the caecum is said to be " usually moderately congested, ecchymotic, and often of a dark slate-grey colour." Clinical evidence is also forthcoming to show that a deficient diet, as experienced in a prisoners of war camp, may produce the same changes in the behaviour of the bowel. Guarinit has studied forty cases of deficiency inanition which developed the condition of chronic intestinal stasis, $x$-ray examina tion showing visceroptosis, distension of the gut, and constipation.

These pathological ehanges in the bowel are associated with an alteration in the adrenaline secretion (McCarrison). Further studies on this subject are being carried out. In the meantime there appears to be sufficient evidence to justify the conclusion that the changes in the bowel associated with chronic intestinal stasis and resulting in pigmentation of the mucosa, interstitial fibrosis, and degeneration of the intrinsic neuro-motor mechanism, may 